\title{
3 Research Soure \\ Controlling of the electrical system through photonic structre via the principle of filtering
}

\section{KP Swain}

Gandhi Institute For Technological Advancement

\section{Subhra Rani Mondal}

Dai Hoc Duy Tan

\section{M.R Nayak}

Biju Patnaik University of Technology

\section{Manikandan Ramachandran}

SASTRA University School of Computing: Shanmugha Arts Science Technology and Research Academy School of Computing

Gopinath Palai ( $\sim$ gpalai28@gmail.com )

Gandhi Institute For Technological Advancement https://orcid.org/0000-0002-9891-493X

\section{Research Article}

Keywords: Photonic filter, VLC, PWE, ATmega 328, ZCD, OOK Signal

Posted Date: June 16th, 2021

DOI: https://doi.org/10.21203/rs.3.rs-176413/v1

License: (c) (i) This work is licensed under a Creative Commons Attribution 4.0 International License.

Read Full License 


\section{Abstract}

The control of the electrical system through a one-dimensional photonic crystal structure is realized in this paper. Here, the electrical system deals with the single-phase induction motor, where the photonic structure is manipulated with the silicon-based monochromatic optical filter. Further, the physics and mathematics of the proposed filter deal with the reflectance characteristics and plane wave expansion method respectively. Moreover, the principle of speed control in the induction motor relies on the simulation outcomes of the photonic filters which communicate the motor through a visible link using a microcontroller along with a ZCD (zero crossing detector) which decides the firing angle of the triggering circuit. Both, Matlab Simulink and physical implementation shows the nitty-gritty of the system which can easily be implemented in real-life application.

\section{Introduction}

Even though photonics research has been accelerating since the last two decades, it is not constrained and confined toa single domain only. Several works related to photonics have been burgeoning and penetrating to the multiple areas vis-à-vis communication, sensing, and networking. Basically, the realization of different types of photonic applications is based on different structures of materials used for fabrication and its several configurations. For example, a Grating structure is one of the frequently used materials for realizing the photonic filters where Silicon and SiO are used alternatively in the literature [1-4]. In all the said literature 64 alternative layers of Silicon (Si) and SiO with two defect positions are implemented to realize the same. In [1], the operating range of filters is the visible range $(400 \mathrm{~nm}$ to $700 \mathrm{~nm}$ ), in [2-3], the range is from $1500 \mathrm{~nm}$ to $1600 \mathrm{~nm}$ whereas the $1300 \mathrm{~nm}$ range is taken in [4]. Instead of using SiO, the air is sandwiched between two Silicon layers in [5] to realize three numbers of DeMUX for signal wavelength $1290 \mathrm{~nm}, 1310 \mathrm{~nm}$, and $1330 \mathrm{~nm}$ by FDTD method where the thickness of Silicon and air is the decisive factor to decide the allowable wavelength. A modified Rowland grating structure is proposed in [6] to realize the optical filters which can be applied to design MUX/ DeMUX, detector array, multiple lasers, etc.

Aside from this, some works of literature [7-11] focus on the optical filters about visible range using 1-D and 2-D photonic crystal. In [7], a 2-D photonic crystal is fabricated using nanoimprint lithography where RAVA (rigorous coupled-wave analysis) is used to study the theoretical properties of the color filters. RGB filters are also discussed in [8] which are based on both index-modulation and periodic modulation type woodpile photonic crystal layers. Three primary monochromatic filters which allow $470 \mathrm{~nm}, 540 \mathrm{~nm}$, and $648 \mathrm{~nm}$ for blue, green, and red spectrum correspondingly in [9] using photonic crystal waveguide where both PWE simulation and finite element method is used to study the dispersion curve and propagation characteristics respectively. In [10], an RGB color filter is implemented using $\mathrm{Si}^{-} \mathrm{ZrO}_{2}-\mathrm{Mn}_{3}{ }^{+} \mathrm{PO}_{4}$ grating in SOI waveguide where FDTD analysis is carried out to study the reflectance characteristics. Using a single patterning process, RGB color filters are developed in [11] where a glass substrate is implemented using nanoimprint lithography and laser annealing process. 
In this research, the generation of monochromatic signals from the one-dimensional photonic structure is realized through the analysis of the one-dimensional Helmotz equation throughplane wave expansion technique. The plane wave expansion is a powerful method to study the reflectance and transmission characteristics of the photonic structure. Moreover, it has certain primacy such as; it is suitable for modal solution problem and is highly used for solving the Eigenvalue problem of photonic structure and also, easy to implement to obtain the band structure and other characteristics. As far as the intrinsic mechanism is concerned, the electric field and the magnetic field are expressed through the Fourier series expansion along with the reciprocal lattice vector, where permittivity plays a vital role. By implementing the PWE technique, a series of photonic filters are proposed to control the speed of an induction motor through the visible link. Aside from these filters, two microcontrollers are implemented at both transmitter and receiver end along with a ZCD (zero-crossing detection) circuit which is working hand to hand with the receiver microcontroller to decide the firing angle of the triggering circuit. At the outset, seven monochromatic filters are designed in visible light range using PWE simulation which allows seven monochromatic color signal wavelengths corresponding to seven colors. The combination of these filter outputs are transmitted as a single bitstream through visible spectrum by the transmitted microcontroller in the form of OOK (on-off keying) signal and the OOK signals are decoded by the receiving microcontroller to decide the firing angle of the triggering circuit which subsequently controls the speed of the motor.Though, the present works deals with the partly theoretical (photonic structure) and partly experimental (electric motor), the novelty lies with the controlling of electrical appliances through photonics. For example, the generation of an exact single (monochromatic) wavelength from the photonic structure is a new result because the outcomes show the generation of a single wavelength in the nanometer range. Here, a single wavelength has been generated without the realization of a broad signal. Further, the monochromatic incident rays which are driven from the LED are used to control the motor with the help of a microcontroller, which could be employed in any electrical control system.

\section{System Overview}

As the structure aims to work in the visible spectrum range $(400-700 \mathrm{~nm})$, so any light source will work perfectly to apply at the first block of the proposed structure which is represented in Fig.1. A dispersive triangular in shape prism is used in this work as the first part of the whole structure to split the visible light spectrum into seven spectral color components such as violet (400-450 nm), blue (450-490 nm), cyan $(490-520 \mathrm{~nm})$, green $(520-560 \mathrm{~nm})$, yellow $(560-635 \mathrm{~nm})$ and red $(635-700 \mathrm{~nm})$. A flint glass is a good candidate to be used here as it possesses a stronger dispersion property than any other glass materials to disband the light. A diffraction grating material may be another choice for this type of work. At the second stage, a combination of seven 1-D photonic monochromatic filters is used here to allow seven monochromatic light signals, one for each seven color band such as $418.1 \mathrm{~nm}, 457.4 \mathrm{~nm}, 499.1$ $\mathrm{nm}, 556.9 \mathrm{~nm}, 588.6 \mathrm{~nm}, 632.5 \mathrm{~nm}, 691.7 \mathrm{~nm}$ for violet, blue, cyan, green, yellow, orange, red respectively. Each filter is realized using (PWE) plane wave expansion method where silicon grating structure is used as background material. The filter is designed by considering 68 alternative layers of SiO and Si where the defect is taken at the $18^{\text {th }}$ and $52^{\text {nd }}$ layer in which $\mathrm{Si}$ is replaced by air as shown in Fig. 2 . The 
different allowable signals in a particular range are selected by varying the width of the SiO (treated as an odd layer) and Si (treated as even layer). For example, the thickness of SiO (143 nm) and Si (42 nm) allows the light signal of wavelength $418.1 \mathrm{~nm}$ for a violet color band of 400 to $450 \mathrm{~nm}$ and at the same time the other signals in the range are rejected. The thickness information for the rest of the color band along with the corresponding allowable signals is given in Table 1.

Table 1: Thickness details of odd and even layers with corresponding allowable signals

\begin{tabular}{|c|c|c|c|c|}
\hline Color & Signal Band & Allowable Signal & Thickness (SiO) & Thickness (Si) \\
\hline Violet & $400-450 \mathrm{~nm}$ & $418.1 \mathrm{~nm}$ & $143 \mathrm{~nm}$ & $42 \mathrm{~nm}$ \\
\hline Blue & $450-490 \mathrm{~nm}$ & $459.4 \mathrm{~nm}$ & $151 \mathrm{~nm}$ & $50 \mathrm{~nm}$ \\
\hline Cyan & $490-520 \mathrm{~nm}$ & $499.1 \mathrm{~nm}$ & $159 \mathrm{~nm}$ & $57 \mathrm{~nm}$ \\
\hline Green & $520-560 \mathrm{~nm}$ & $556.5 \mathrm{~nm}$ & $157 \mathrm{~nm}$ & $61 \mathrm{~nm}$ \\
\hline Yellow & $560-595 \mathrm{~nm}$ & $588.6 \mathrm{~nm}$ & $157.8 \mathrm{~nm}$ & $71.5 \mathrm{~nm}$ \\
\hline Orange & $595-640 \mathrm{~nm}$ & $632.5 \mathrm{~nm}$ & $173 \mathrm{~nm}$ & $74 \mathrm{~nm}$ \\
\hline Red & $640-700 \mathrm{~nm}$ & $691.7 \mathrm{~nm}$ & $193.2 \mathrm{~nm}$ & $77.5 \mathrm{~nm}$ \\
\hline
\end{tabular}

Seven color sensors with a microcontroller (ATmega 328 of Arduino) are used in the subsequent stage to detect the seven corresponding light signals and to convert the equivalent electrical signals into binary data streams and afterward, the corresponding OOK signals. In this process, as seven optical signals are coming out from the filters so, total $2^{7}=128$ combinations ('0000000' to '1111111') are possible by controlling the signals manually and each combination represents an individual data steam which is converted into corresponding OOK signal by the microcontroller. OOK (on-off keying) is the simplest form of ASK (amplitude shift keying) modulation scheme which represents the binary ' 1 ' as the presence of a signal and binary ' $O$ ' as the absence of signal. At last, the microcontroller drives a LED according to OOK signals to communicate with the receiver through the VLC link.

Fig. 3 shows the circuit diagram of the transmitting microcontroller (ATmega 328) which consists of seven photodetectors to convert the optical signals coming from the optical filters into electrical voltages. The equivalent voltages were then fed into the microcontroller pins (PD2 to PD7 and PB0). A program is written to convert these parallel data into binary data streams with start and stop bits for transmitting serially through a transmitting LED connected at pin PC2.

About the receiver part, it is comprising of three major sub-units like microcontroller with a photodetector to receive the OOK signal which is coming through VLC link from transmitter end, motor driver circuit with motor and ZCD (zero crossing detector) to detect the zero-crossing time of ac input voltage as shown in the circuit diagram Fig. 4. The OOK signals are first detected by the photodetector whose output is connected to PD3 pin of ATmega 328 microcontroller for converting the binary streams into a decimal voltage which in turn mapped into the firing angle of 0 to 180 degree of gate voltage (AC) of Triac to control the speed of the motor. Not only is the firing angle, the microcontrolleris also used to detect the time of crossing zero reference line of AC waveform with the help of a zero-crossing detector circuit. The signal from ZCD is connected to the microcontroller which is used to generate software interrupt when the rising edge is detected at the input pin of the microcontroller. From that instant, a time delay can be introduced within a $9 \mathrm{~ms}$ time window to achieve a phase from 0 to 180 degrees. Considering the turn-on 
time of Triac approximately $1 \mathrm{~ms}$ and total time is $10 \mathrm{~ms}$ for a.c input (50 Hz), so $9 \mathrm{~ms}(10 \mathrm{~ms}-1 \mathrm{~ms})$ is taken into account to map the phase angle from 0 to 180 degrees. Here, the microcontroller generates a software interrupt for each half cycle of a.c input to calculate the time delay for determining the phase angle. Here, the choice of the off the shelf components including ICs and micro-controller is illustrated below.

Microchip ATmega 328 is a general-purpose $16 \mathrm{MHz}$, 8-bit RISC microcontroller of AVR series which has three timers (two 8-bit and one 16-bit), ADC (10 bit), 32 KB of flash memory, serial port (one USART, two $\mathrm{SPI}$, and one I2C) and PWM (six pins). This microcontroller is mostly used as the prime component in the Arduino Development board. In this work, Arduino is used as the main components while performing simulation using Matlab Simulink and ATmega328 microcontroller with its associated circuits are used in the hardware realization where the speed of an a.c motor is controlled. Among the other major components, a Triac BT136 $(600 \mathrm{v}, 5 \mathrm{~A})$ is used to drive the motor depending upon the firing angle of input a.c input voltage which is decided by the receiver microcontroller and a ZCD (zero-crossing detection) circuit. MOC3021 is an opto-isolator used as a driver for BT136 as merely microcontroller output is not sufficient enough to drive the Triac. Opto-coupler (MOC3021) is used in the ZCD circuit to transfer the electrical energy through light by making isolation between input and output. Initially, the receiver photodetector receives the OOK signal which is transmitted by the transmitter and these OOK signals decide the equivalent firing angle of Triac by the receiver microcontroller.

To recapitulate, the setup consists of two microcontrollers; a transmitting microcontroller for transmitting the optical signals coming from the photonic filters as OOK signals and a receiving microcontroller to receive the OOK signals. The OOK signals are then converted into equivalent decimal value by the microcontroller which is to be mapped into a 0 to 180degrees phase angle of Gate voltage of Traic. Again, with the help of ZCD, the required time delay is calculated for each half cycle of AC input of the triggering circuit for controlling the speed of the induction motor. For example;

Case-1: For OOK signals '0000000' is equal to ' 0 ' in decimal at the receiver side which refers to 0 degrees of phase angle where the motor will rotate at full speed as there is no firing angle control.

Case-2: For OOK signals ' 1111111 ' is equal to ' 127 ' in decimal at the receiver side which refers to 180 degrees of phase angle where the motor is stopped as Triac will not turn ON.

Case-3: For OOK signals ' 1000000 ' is equal to ' 64 ' in decimal at the receiver side which refers to 90 degrees of phase angle and in this case, the motor will rotate by half of the maximum speed.

\section{Results And Implementation}

This section is divided into three sub-sections; PWE simulation to analyze the reflectance curve of the filter characteristics, Matlab Simulink simulation for hardware realization, and physical implementation to verify the receiver model practically. 


\section{PWE Simulation}

PWE simulation is applied to realize the reflectance characteristics of all seven monochromatic color filters. A Silicon grating structure with a defect at two positions is used for the simulation process for all the filters with different thicknesses of $\mathrm{SiO}$ and $\mathrm{Si}$ for corresponding allowable signals. Fig. 5 (a-g) shows the reflectance curve for $418.1 \mathrm{~nm}, 459.4 \mathrm{~nm}, 499.1 \mathrm{~nm}, 556.5 \mathrm{~nm}, 588.6 \mathrm{~nm}, 632.5 \mathrm{~nm}, 691.7 \mathrm{~nm}$ respectively. In the figures, the vertical axis represents the reflectance in Arb. Unit and horizontal axis is used to represent the range of the signals in the nm scale. The thickness of SiO and $\mathrm{Si}$ is taken as 143 $\mathrm{nm}$ and $42 \mathrm{~nm}$ respectively to allow the signal wavelength of $418.1 \mathrm{~nm}$ as shown in Fig. 3 (a). It is observed that the reflectance is zero for the above-said wavelength and is equal to one for other signals within the particular color band. This also reveals that if the violet color band of light is incident on the filter, then only $418.1 \mathrm{~nm}$ of the light signal is passed through the filter structure while all other signals are completely reflected. Similarly, Fig. $5(b-g)$ represents the reflectance characteristics of the corresponding filters.

\section{Matlab Simulink}

Matlab Simulink is implemented for physical validationby considering three cases; zero speed, half of the maximum speed, and maximum speed.As, VLC link cannot be realized in Matlab Simulink, so the wired connection has been made between transmitter and receiver. PPC (phase control pulse) method is implemented for speed control of A.C. motor which is performed by the microcontroller at both transmitter and receiver end. In the beginning, an optical band separator is used to separate the different color bands like violet, blue, cyan, green, yellow, orange, red in the range of $400 \mathrm{~nm}$ to $700 \mathrm{~nm}$. Then the individual color band of light is incident on the photonic filters which is the combination of seven monochromatic color filters. These filters are used to pass the seven monochromatic color signals of bandwidth $418.1 \mathrm{~nm}, 459.4 \mathrm{~nm}, 499.1 \mathrm{~nm}, 556.5 \mathrm{~nm}, 588.6 \mathrm{~nm}, 632.5 \mathrm{~nm}, 691.7 \mathrm{~nm}$ for the seven corresponding colors. These signals are sensed by the photodetectorindividually and control by seven switches from D6 to D0 which result in 128 combinations from '0000000' to '1111111'. All these combinations are mapped into 0 to 180 degrees of the phase angle of the half cycle of the input source.So this mapping of phase angle is essentially used to change the average voltages of sine wave across the motor terminal which resultsin the speed control as shown in table 2.

Among 128 numbers of combinations, only three cases are taken; '0000000', '1000000', and '1111111'. Fig. 6(a) shows the simulation for the input combination of '0000000' which is used to operate the motor at full speed. Here, the combination '0000000' is mapped into zero degrees, so there is no control of the firing angle. This indicates the full input voltage is available at the motor terminal and the motor is rotating with maximum speed.

Similarly, the status of the motor is specified in Fig. 6 (b) and (c) for the input combination of ' 1000000 ' and ' 1111111 ' respectively. Here, the motor is rotating in half of the maximum speed for ' 1000000 ' and in minimum (stop) speed for ' 1111111 ' which is illustrated by a tachometer with a pointer. 
Table-2: Speed of the motor with corresponding input combination

\begin{tabular}{|c|c|c|c|c|c|c|c|c|}
\hline D6 & D5 & D4 & D3 & D2 & D1 & D0 & FiringAngle & Speed of Motor \\
\hline 0 & 0 & 0 & 0 & 0 & 0 & 0 & Minimum & Max (full speed) \\
\hline i & $\mathrm{i}$ & $\mathrm{i}$ & $\mathrm{i}$ & $\mathrm{i}$ & $\mathrm{i}$ & $\mathrm{|}$ & & \\
\hline 1 & 0 & 0 & 0 & 0 & 0 & 0 & Half & Speed is half \\
\hline $\mathrm{i}$ & $\mathrm{i}$ & $\mathrm{i}$ & $\mathrm{i}$ & $\mathrm{i}$ & $\mathrm{|}$ & $\mathrm{|}$ & & \\
\hline 1 & 1 & 1 & 1 & 1 & 1 & 1 & Maximum & Min (Stop) \\
\hline
\end{tabular}

In this experiment, as we are getting nearly 128 combinations of binary data streams, so it can be implemented in any similar type of controlling application. For example, various movements of the robot can be controlled with a different combination of input data. The different sensors can be also deployed on the robot which can be mapped into different combinations of data to design a full-fledged optical control robot.

\section{Physical Implementation}

The physical implementation is carried out to test the receiver model functionality whether the speed control is achieved by binary bits or not. Fig. 7(a) represents the general setup which consists of a DSO for the representation of firing angle of triggering circuit with respect to input source of a.c voltage, ZCD, receiver circuit with a.c adapter, and induction motor with a simple blade. Here, three push button switches are used for the generation of the different binary data streams which characterizes as a transmitter.

When the model is switched ON, the transmitter switches represent ' 000 ' with minimum firing angle as shown in Fig. 7 (b) which permits the full ac voltage across the motor, and consequently motor is rotated at full speed. In another case as in Fig. 7 (c), for any intermittent combination of transmitter switches, the firing angle is started late so, the motor speed is reduced to nearly half of the full speed.

\section{Conclusion}

In this paper, the speed of the single-phase induction motor is controlled by the monochromatic photonic filters through the visible link where reflectance characteristics are the backbone of the optical filters, and plane wave expansion is the foundation to generalize the reflection loss of the structure. Aside from this, a zero-crossing detector with a microcontroller is also a mainstay to reveal such an application. Finally, the present work affirms that the proposed research would bring a new paradigm to realize the optical control electrical system.

\section{References}

1. K.P. Swain, G. Palai, J.K. Moharana, Design and implementation of opto-electro decoder using photonic structure: A new application of Li-fi vis-a-vis optical embedded system, Optik - International Journal for Light and Electron Optics, Vol. 178, pp. 658-663, 2019 
2. K.P. Swain, G. Palai, J.K. Moharana, Analysis for '101' channels of MUX/DEMUX using grating SOI Structure at sub nanometer scale, Optik, Vol. 129, pp.78-82, 2017

3. K.P. Swain, G. Palai, J.K. Moharana, Optical filter based electrical control vis-à-vis cloud data: A new hybrid optoelectronics device for embedded application, Optik - International Journal for Light and Electron Optics, Vol. 178, pp. 964-969, 2019

4. K.P. Swain, G. Palai, Photonic structure for embedded application: Realization of optical filter based locking system, Optik - International Journal for Light and Electron Optics, Vol. 169, pp. 344-349, 2018

5. J.S.N. Achary, G. Palai, S.K. Tripathy, Realization of an optical demultiplexor using the combination of filter and finite difference time domain approach, Optik, Vol. 150 pp.94-98, 2017

6. Jia-Xian Wang, Jing Zhao, Weibin Qiu, Zhili Lin, Yixin Huang, Houbo Chen, Pingping Qiu, Novel optical filters based on curved grating structure, Optics Communications, Vol. 387, pp. 61-64, 2017

7. Eun-Hyoung Cho, Hae-Sung Kim, Byoung-Ho Cheong, Prudnikov Oleg, Wenxu, Xianyua, Jin-Seung Sohn, Dong-Joon Ma, Hwan-Young Choi, No-Cheol Park, Young-Pil Park, Two-dimensional photonic crystal color filter development, Optics express, Vol. 17, no. 10, pp.8621- 8629, 2009

8. Hee-Jin Choi, Sujin Choi, Young-Eun Yoo, Eun-chae Jeon, Yun Yi, Shinwoong Park, Doo-Sun Choi, Hwi Kim, Transmission-type photonic crystal structures for color filters, Optics express, Vol. 21,no. 15, pp. 18317-24, 2013

9. Brahm Raj Singh, Swati Rawal, R. K. Sinha, Photonic crystal-based RGB primary colour optical, Filter, Journal of Modern Optics, Vol. 63, no. 14, pp. 1391-1396, 2016

10. Ramanpreet Kaur, Neetu Sharma, Jaspreet Kaur, RGB color filter based on triple layer high contrast grating in SOI waveguide, J. Optics, Vol. 46, no. 3, pp. 320-323, 2017

11. Eun-Hyoung Cho, Hae-Sung Kim, Jin-Seung Sohn, Chang-Youl Moon, No-Cheol Park, Young-Pil Park, Nanoimprinted photonic crystal color filters for solar-powered reflective displays, Optics express, Vol. 18, no.26, pp. 27712-22, 2010

\section{Figures}




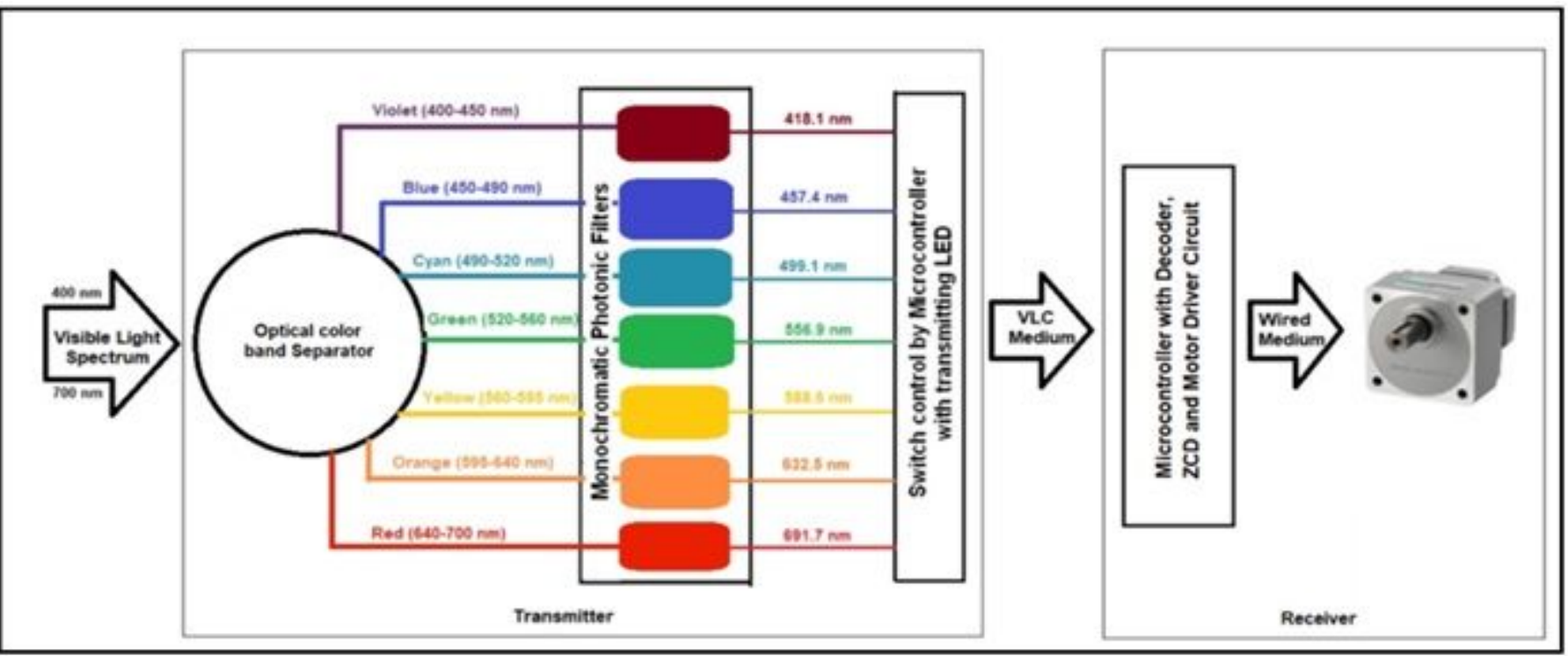

Figure 1

Graphical overview of the proposed structure

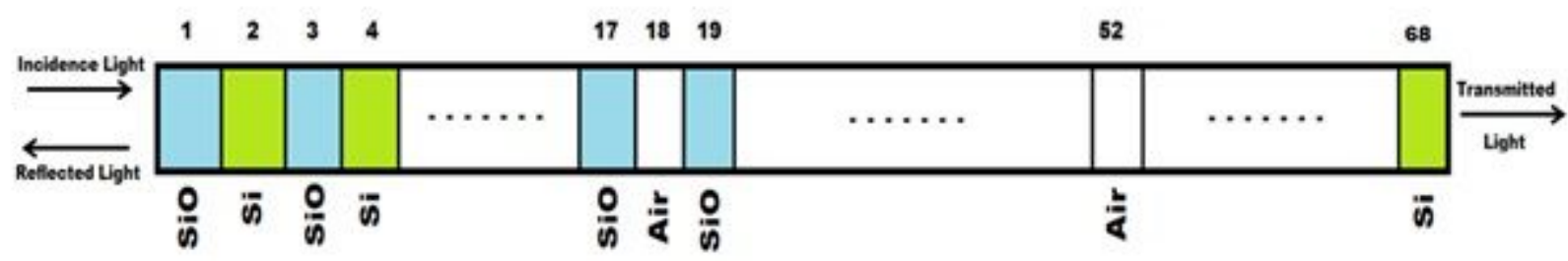

Figure 2

Silicon grating structure of the 1-D photonic filter 


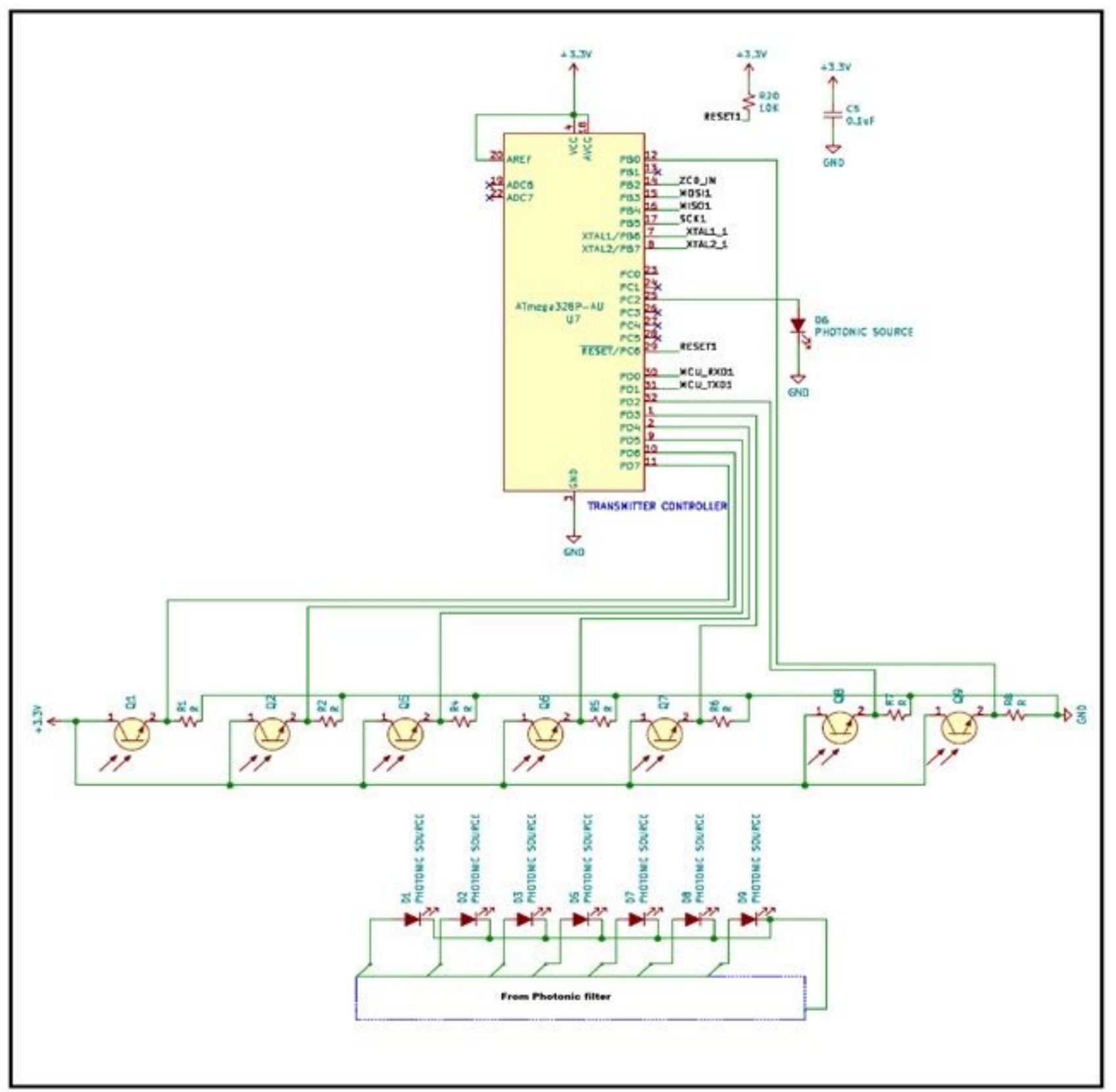

Figure 3

Circuit diagram of transmitting microcontroller with associated circuit 


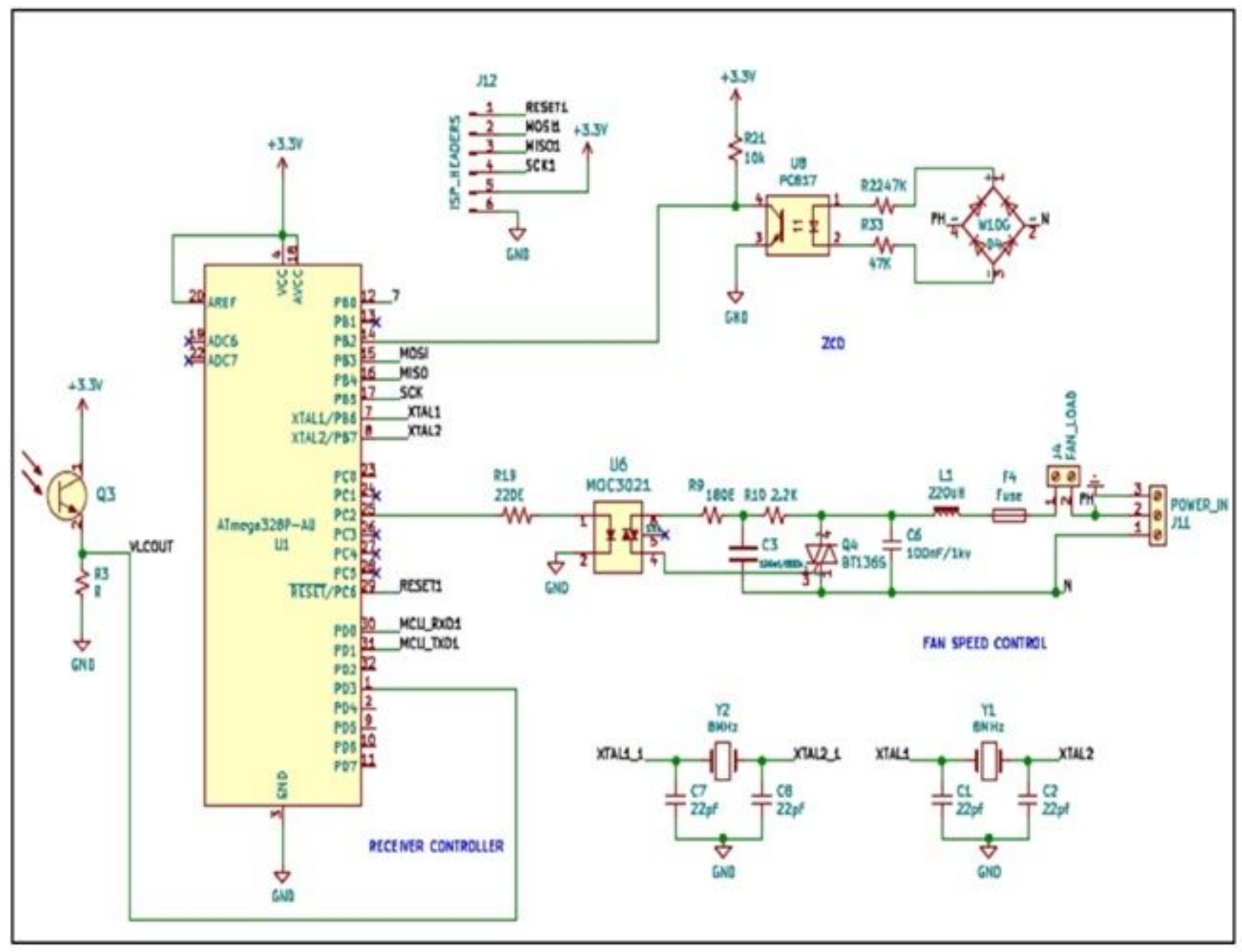

Figure 4

Circuit diagram of the receiver circuit 

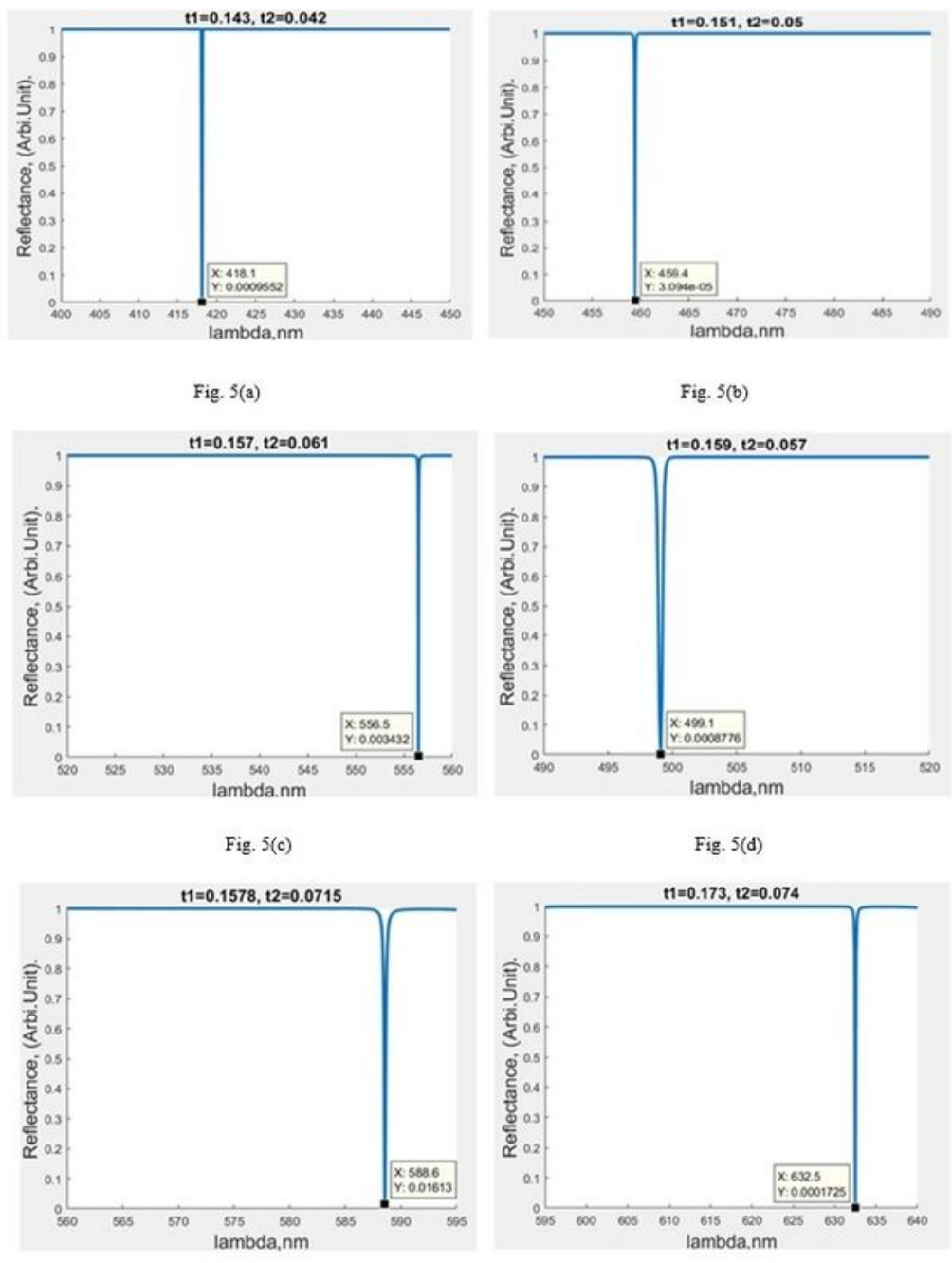

Fig. 5(e)

Fig. 5(f)

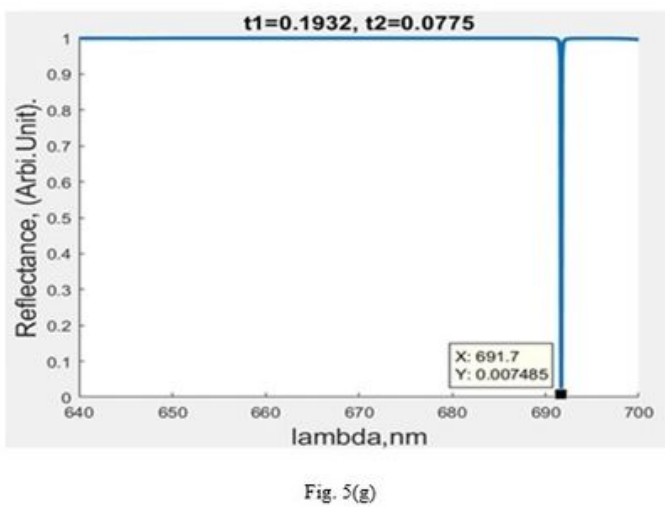

\section{Figure 5}

(a-g). Reflectance characteristic curves for different monochromatic color filters obtained by PWE simulation 

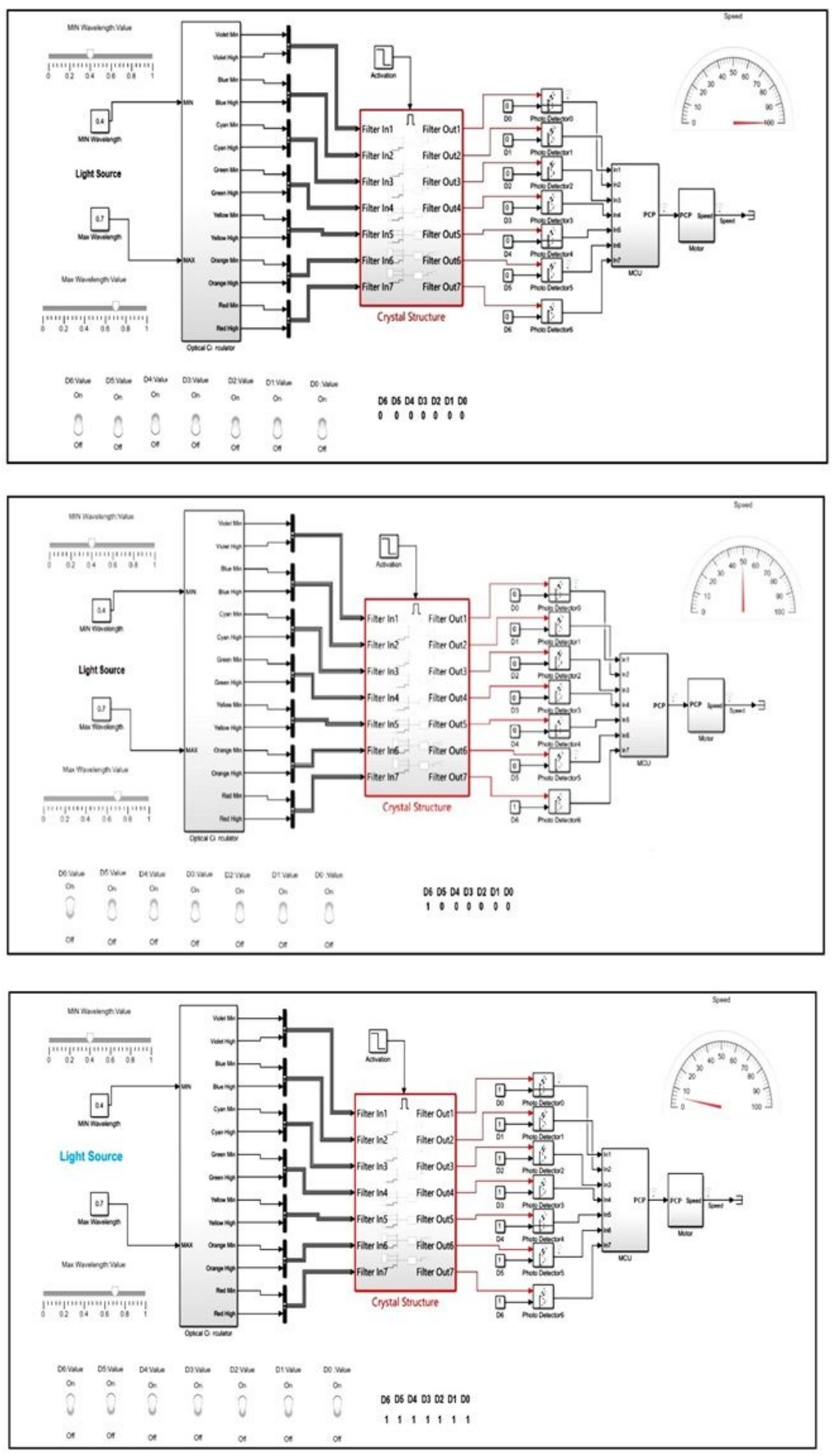

\section{Figure 6}

(a). Simulink output for the input combination of ' 0000000 ' where the speed is maximum as the full input voltage is available at motor terminal (b). Simulink output for the input combination of ' 1000000 ' where the speed is medium as phase angle is controlled from 90 degrees of the input phase (c). Simulink output for the input combination of ' 1111111 ' where the speed in minimum as firing angle is started from 180 degrees of the input phase 

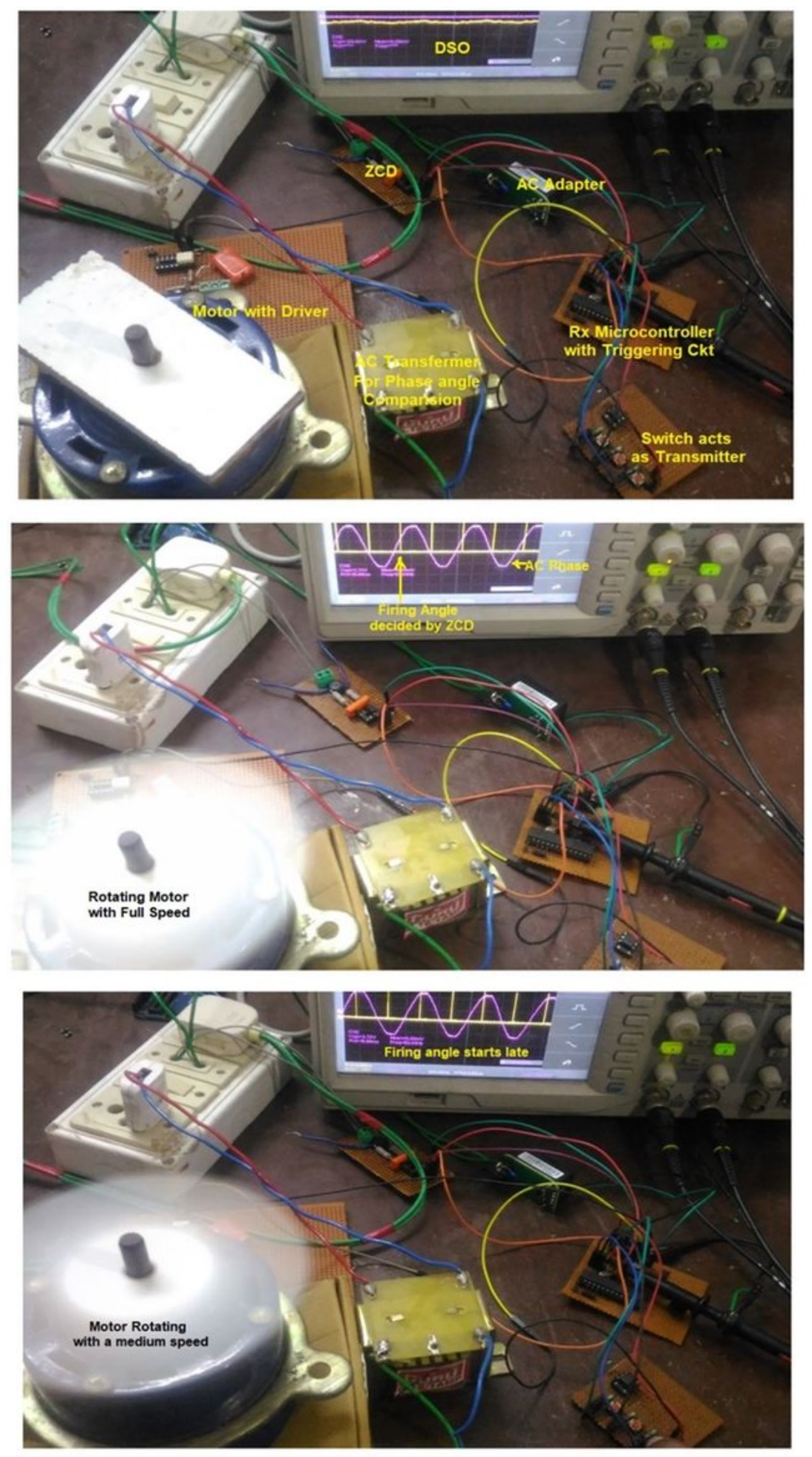

\section{Figure 7}

(a). The physical implementation of the proposed model (b). Motor with full speed as firing angle start immediately (c). Motor with full speed as firing angle start late 